\title{
Determinação da Distribuição de Funcionalidade de HTPB e Verificação de sua Influência no Comportamento Mecânico de Poliuretano Utilizado em Motor-Foguete
}

\author{
Vera L. Lourenço, Aparecida M. Kawamoto, Jairo Sciamareli, Luis C. Rezende, Darci C. Pires, Marta F. K. Takahashi, \\ Agda V. Berdugo, Sandra M. Cruz, Rita C.L. Dutra \\ Divisão de Química, IAE, CTA, SP
}

Bluma G. Soares

Instituto de Macromoléculas Professora Eloisa Mano, UFRJ

Resumo: Um método SEC com dupla detecção para determinação de distribuição de funcionalidade de resina polibutadiênica hidroxilada HTPB foi desenvolvido, verificando-se a influência desse parâmetro no comportamento mecânico de poliuretano utilizado em motor-foguete fabricado no Centro Técnico Aeroespacial (CTA). O método mostrou-se sensível a pequenas diferenças de funcionalidade e sua distribuição podendo ser utilizado para a caracterização mais completa da resina. Ensaios de tração realizados em amostras de PU sem carga refletiram pequenas diferenças de distribuição de funcionalidade existentes entre os lotes de polibutadieno hidroxilado.

Palavras-chave: Resina polibutadiênica hidroxilada (HTPB), funcionalidade, poliuretano, propriedades mecânicas.

\section{Determination of Functionality Distribution of HTPB and Evaluation of its Influence in the Mechanical Behaviour of Polyurethane Used in Rocket Cases}

\begin{abstract}
A size exclusion chromatography (SEC) method, with double detection, was developed to determine the functionality distribution of hydroxyl polybutadiene (HTPB) resin, evaluating the influence of that parameter on the mechanical behavior of HTPB polyurethane used as binder in the rocket motors manufactured at Centro Técnico Aeroespacial (CTA). The method showed to be sensitive to small differences in the functionality distribution of the HTPB resin and, thus, can be used for a more complete characterization of the resin. Tensile tests performed on HTPB polyurethane reflected the small differences observed in the functionality distribution of three batches of HTPB.
\end{abstract}

Keywords: Hydroxyl polybutadiene resin (HTPB), functionality, solid propellant, mechanical behaviour.

\section{Introdução}

Como é conhecido, HTPB é um importante ligante polimérico combustível em propelentes sólidos ${ }^{[1,2]} \mathrm{em}$ função de suas excelentes propriedades mecânicas e balísticas, sendo que dentre os mais importantes parâmetros característicos do HTPB está a sua distribuição de funcionalidade, ou seja, número de grupos reativos por molécu$\mathrm{la}^{[1]}$. O polímero, preparado por polimerização via radical livre, não é essencialmente telequélico e possui um larga distribuição de massa molar e funcionalidade ${ }^{[1,2]}$.

Quando curado com um isocianato difuncional, como tolueno diisocianato (TDI), moléculas não funcionais não participam da reação com isocianato, enquanto que as monofuncionais agem como terminadores de cadeia, as difuncionais como extensores de cadeia e as tri e polifuncionais como formadores de ligação cruzada $^{[3]}$. Então, as quantidades relativas destas moléculas influenci- am as propriedades mecânicas do propelente curado ${ }^{[1-3]}$. Sendo assim, o desenvolvimento de metodologias para a determinação da distribuição de funcionalidade de HTPB, associado à verificação da influência desse parâmetro no comportamento mecânico do propelente, apresenta-se como uma caracterização importante em projetos aeroespaciais.

Várias tentativas de caracterizar a distribuição de funcionalidade de HTPB têm sido relatadas na literatura ${ }^{[1-12]}$.

Métodos relacionando a distribuição de massa molar e a distribuição de funcionalidade envolveram três principais alternativas. Na primeira, a amostra foi fracionada em função da funcionalidade através de cromatografia de adsorção em coluna ${ }^{[1,4-6]}$, seguida pela determinação da massa molar por Osmometria de Pressão de Vapor (VPO ${ }^{[5]}$ ou Cromatografia de Exclusão por Tamanho (SEC) ${ }^{[4,6]} \mathrm{e}$ da massa equivalente das frações por espectroscopia no infravermelho ${ }^{[4-6]}$. Na segunda, a amostra foi fracionada em função da massa molar por SEC e, em seguida, a

Autor para correspondência: Vera L.Lourenço, AQI-CTA/IAE, Pr. Mal Eduardo Gomes 50, CEP: 12228-904, São José dos Campos, SP. E-mail:vlucia@iae.cta.br. 
funcionalidade de cada fração foi determinada por Cromatografia de Camada Fina (TLC) $)^{[7]}$.

Uma terceira alternativa foi sugerida por Anderson ${ }^{[8,9]}$, na qual a amostra é derivatizada, ou seja, reagida com um cromóforo absorvente na região do ultravioleta (UV), como o isocianato de fenila, de forma que o grupo cromóforo seja introduzido no grupo funcional. O produto, ou derivado, é analisado por SEC com dois detetores, sendo um de índice de refração (RI) e outro de UV. O sinal do RI é utilizado para calcular a massa molar e a relação entre os dois sinais é utilizada para calcular a funcionalidade, permitindo a construção da curva de funcionalidade $\mathrm{x}$ massa molar. Este método foi denominado SEC dupla detecção. Ceccaldi ${ }^{[10]}$ fracionou por cromatografia de adsorção o HTPB e derivatizou as frações com cloreto de 3,5-dinitrobenzoíla, aplicando em seguida o método SEC dupla detecção, obtendo resultado igual ao sem fracionamento.

O fracionamento do HTPB derivatizado por SEC preparativo $^{[3]}$ ou por cromatografia de adsorção ${ }^{[1]}$, seguido por SEC dupla detecção para caracterizar as frações também levou aos mesmos resultados obtidos sem o pré-fracionamento.

Desde então, o método SEC dupla detecção tem sido usado para caracterizar HTPB de diferentes procedências e para avaliar a influência da distribuição de funcionalidade nas propriedades mecânicas de propelentes ${ }^{[2,3,11,12]}$.

Sendo assim, neste trabalho é relatada a aplicação deste método ao HTPB Liquiflex-P, empregado na produção de propelentes no CTA, utilizando derivados éster de isocianato de fenila, conforme o método citado por Anderson ${ }^{[8,9]}$. A influência da distribuição de funcionalidade no comportamento mecânico do propelente sólido foi avaliada com a realização de ensaios de tração em formulações de poliuretano sem carga, que constitui a matriz polimérica das formulações de elastômeros e propelentes desenvolvidas no CTA, constituído de HTPB, diisocianato de isoforona (IPDI) como agente de cura e acetilacetonato férrico como catalisador de cura, de modo a permitir avaliar as propriedades da matriz polimérica, sem a interferência de cargas e outros aditivos.

\section{Experimental}

\section{Características dos materiais utilizados}

A Tabela 1 mostra os valores de índice de hidroxilas $([\mathrm{OH}] \mathrm{meq} / \mathrm{g})$ e massa molar numérica média $(\mathrm{Mn})$ obtida por osmometria de pressão de vapor (VPO) dos lotes de HTPB Liquiflex P da Petroflex, utilizados. Na obtenção dos derivados foi usado isocianato de fenila Merck, pureza 97,9\% (MM $=119 \mathrm{~g} / \mathrm{mol})$. Foram usados $4,2 \mathrm{meq}$ de NCO, pois a reação se passa consumindo 2 mols de NCO para $1 \mathrm{~mol} \mathrm{de} \mathrm{OH}$.

\section{Síntese dos derivados}

A síntese dos derivados éster da resina HTPB foi realizada segundo um procedimento similar ao utilizado por Anderson ${ }^{[8]}$, porém, com algumas alterações: os reagentes foram utilizados como recebidos, reagidos a $70{ }^{\circ} \mathrm{C}$ por $9 \mathrm{~h}$, o NCO não reagido foi removido por extração em clorofór-
Tabela 1. Características das resinas utilizadas na síntese dos derivados analisados por SEC

\begin{tabular}{cccc}
\hline HTPB & $\begin{array}{c}\text { [OH] } \\
\mathbf{m e q} / \mathbf{g}\end{array}$ & $\begin{array}{c}\text { Mn(VPO) } \\
(\mathbf{g} / \mathbf{m o l})\end{array}$ & Amostra \\
\hline $\begin{array}{c}\text { Liquiflex-P Lote } \\
\text { 73/97 tb 01 }\end{array}$ & 0,73 & 2870 & HTPB/ISOFEN 98 \\
$\begin{array}{c}\text { Liquiflex-P Lote } \\
\text { 55/98 tb:08+tb:10 }\end{array}$ & $0,75+0,74$ & 2870 & HTPB/ISOFEN 99 \\
$\begin{array}{c}\text { Liquiflex-P Lote } \\
\text { 01/98 tb:14 }\end{array}$ & 0,69 & 2825 & HTPB-ISO/01-2000 \\
$\begin{array}{c}\text { Liquiflex-P Lote } \\
\text { 01/98 tb:14 }\end{array}$ & 0,69 & 2825 & HTPB-ISO/02-2000 \\
$\begin{array}{c}\text { Liquiflex-P Lote } \\
\text { 76/97 tb:06 }\end{array}$ & 0,73 & 2863 & HTPB-ISO/03-2000 \\
$\begin{array}{c}\text { Liquiflex-P Lote } \\
\text { 76/97 tb:06 }\end{array}$ & 0,73 & 2863 & HTPB-ISO/04-2000 \\
$\begin{array}{c}\text { Liquiflex-P Lote } \\
\text { 73/97 tb:10 }\end{array}$ & 0,76 & 2773 & HTPB-ISO/05-2000 \\
$\begin{array}{c}\text { Liquiflex-P Lote } \\
\text { 73/97 tb:10 }\end{array}$ & 0,76 & 2773 & HTPB-ISO/06-2000 \\
\hline
\end{tabular}

mio, e este removido com solução aquosa de $\mathrm{NaHSO}_{3}$ a $1,5 \%$. O produto foi caracterizado por FT-IR, sendo que todas as amostras mostraram absorções de ligações ésteres-uretanas, provenientes da reação $\mathrm{OH} / \mathrm{NCO}$ dos materiais de partida.

\section{Análise cromatográfica/tratamento de dados}

As análises SEC foram realizadas no Instituto de Macromoléculas (IMA/UFRJ) utilizando: cromatógrafo Waters 610 equipado com detector de ultravioleta 410 do tipo "diode array" (254nm) e detector de índice de refração 910 colocados em série, e estação de aquisição e tratamento de dados Máxima 800; colunas Waters ultrastyragel, colocadas em série, sendo uma de porosidade 500 e 2 lineares; a calibração foi feita com padrões de poliestireno de várias massas molares; solvente tetrahidrofurano Merck, vazão 1,00 $\mathrm{mL} / \mathrm{min}$; concentração da amostra $15 \mathrm{mg} / 2 \mathrm{~mL}$; volume injetado: $100 \mathrm{~mL}$; temperatura do forno, injetor e detector $35^{\circ} \mathrm{C}$. As amostras foram dissolvidas em THF, filtradas em filtro de $0,45 \mu \mathrm{m}$ e injetadas no cromatógrafo.

Os sinais de ambos os detetores foram armazenados e tratados com ajuda do programa Máxima 820, que forneceu as curvas de calibração para cada detector, obtidas simultaneamente para os mesmos padrões, os cromatogramas, as massas molares $\overline{\mathrm{M}}_{\mathrm{n}}$ e $\overline{\mathrm{M}}_{\mathrm{w}}$ e a polidispersão das amostras.

Cada cromatograma foi fatiado, pelo programa, em intervalos de 30s, sendo obtida uma tabela onde o tempo de retenção foi relacionado à massa molar calculada em relação à curva de calibração, à altura e à área do pico, para cada detector. O cálculo da funcionalidade requer que os sinais UV e RI sejam tomados em pontos equivalentes ${ }^{[3,8,10]}$. Como o cromatógrafo calculou $\overline{\mathrm{M}}_{\mathrm{n}}$ e $\overline{\mathrm{M}}_{\mathrm{w}}$ para os dois detectores, não foi necessário descontar o tempo para a amostra passar de um para outro, sendo suficiente tomar os sinais nas mesmas $\overline{\mathrm{M}}_{\mathrm{n}}$. No entanto, o início e o fim do pico foram definidos automaticamente pelo 
programa Máxima 820, de acordo com a linha base traçada automaticamente dentro do intervalo de tempo dado pelo operador, ou seja, este não tem controle sobre o intervalo onde é feita a integração e as listagens de MM e tempo de retenção, tr, dos dois setores não coincidentes. Para contornar este obstáculo, os cromatogramas obtidos foram transcritos para uma planilha de cálculo, relacionando a massa molar obtida a partir das curvas de calibração (MM x tr) e o valor dos sinais (área) RI e UV. Isto permitiu a superposição dos dois sinais e obtenção de valores dos sinais na mesma massa molar.

A funcionalidade de cada fração $\left(f_{i}\right)$ foi calculada pela equação $1^{[8,10]}$, onde:

$\mathrm{M}_{\mathrm{i}}$ é Mn obtido do cromatograma RI no tempo $\operatorname{tr}_{\mathrm{i}}$,

$v_{\mathrm{i}}$ é a intensidade (área) do cromatograma RI equivalente a $\mathrm{M}_{\mathrm{i}}$,

$\varepsilon_{\mathrm{i}}$ é a intensidade (área) do cromatograma UV equivalente a $\mathrm{M}_{\mathrm{i}}$,

$\tau$ é o teor dos grupos funcionais em eq./g determinado por dosagem química do HTPB não-derivatizado multiplicado por Mn (Tabela 1).

$$
f_{i}=\frac{\tau \cdot \varepsilon_{i} M_{i}}{v_{i}} \cdot \frac{\sum_{1}^{i} v_{i}}{\sum_{i}^{i} \varepsilon_{i}}
$$

As curvas de distribuição de funcionalidade foram obtidas traçando-se $\mathrm{f}_{\mathrm{i}} \times \mathrm{M}_{\mathrm{i}}$. As curvas de distribuição de massa molar em função da concentração mássica no detetor RI (equação 2) e da concentração de distribuição cumulativa (equação 3 ) também foram traçadas.

$$
\begin{aligned}
& \mathrm{I}_{\mathrm{i}}=\frac{\mathrm{v}_{\mathrm{i}} \cdot 100}{\sum_{1}^{\mathrm{n}} \mathrm{v}_{\mathrm{i}}} \\
& \mathrm{L}_{\mathrm{i}}=\sum_{1}^{i}\left(\frac{\mathrm{v}_{\mathrm{i}} \cdot 100}{\sum_{1}^{\mathrm{n}} \mathrm{v}_{\mathrm{i}}}\right)
\end{aligned}
$$

\section{Procedimento de preparação do poliuretano (PU)}

Amostras de poliuretano referentes a três lotes de HTPB foram preparadas conforme procedimento descrito na seqüência e as respectivas composições são apresentadas na Tabela 2. Devido aos diferentes índices de hidroxilas, para cada amostra de HTPB foi usada uma quantidade diferente de IPDI, suficiente para se obter razão $\mathrm{NCO}: \mathrm{OH}$ igual a 1,0. Cada formulação contém cerca de $70 \mathrm{ppm}$ do catalisador de cura acetilacetonato férrico. $\mathrm{O}$ catalisador foi pesado e adicionado, com auxílio de $1 \mathrm{ml}$ de diclorometano a $100 \mathrm{~g}$ de HTPB; após aquecimento a $50{ }^{\circ} \mathrm{C}$ sob vácuo, para eliminação das bolhas de ar, foi adicionado o IPDI, misturado à resina sob vácuo e carregada a matriz (70-75 g) que foi mantida por 8 dias em estufa a $50{ }^{\circ} \mathrm{C}$ para completar a cura.
Tabela 2. Composição dos PU

\begin{tabular}{lccc}
\hline \multicolumn{1}{c}{ HTPB } & $\begin{array}{c}\text { Lote 01/98, } \\
\text { Tb 14 }\end{array}$ & $\begin{array}{c}\text { Lote 76/97, } \\
\text { Tb 06 }\end{array}$ & $\begin{array}{c}\text { Lote 73/97, } \\
\text { Tb 10 }\end{array}$ \\
\hline$[\mathrm{OH}](\mathrm{meq} / \mathrm{g})$ & 0,69 & 0,73 & 0,76 \\
HTPB $(\mathrm{g})$ & 100,0 & 100,0 & 100,0 \\
Catalisador $(\mathrm{g})$ & 0,0077 & 0,0077 & 0,0077 \\
IPDI (g) & 7,66 & 8,11 & 8,44 \\
NCO:OH & 1,0 & 1,0 & 1,0 \\
\hline
\end{tabular}

Determinação de propriedades mecânicas do PU: Ensaios de tração

Após a cura, os discos de PU foram encaminhados para o Laboratório de Propriedades Mecânicas (IAE/AQI-PPM) para preparação dos corpos-de-prova para os ensaios de tração. Estes foram realizados na máquina de ensaios universal Instron, modelo 1130 , a $500 \mathrm{~mm} / \mathrm{min}$ e na temperatura ambiente, conforme norma ASTM D-412. Os dados obtidos mais relevantes foram a tensão de ruptura $\left(\sigma_{\mathrm{r}}\right)$ e o alongamento na ruptura $\left(\varepsilon_{\mathrm{r}}\right)$.

\section{Resultados e Discussão}

\section{Distribuição de funcionalidade do HTPB}

As Figuras 1 a 4 mostram as curvas de concentração mássica (\%xMM) no detetor RI e da concentração cumulativa

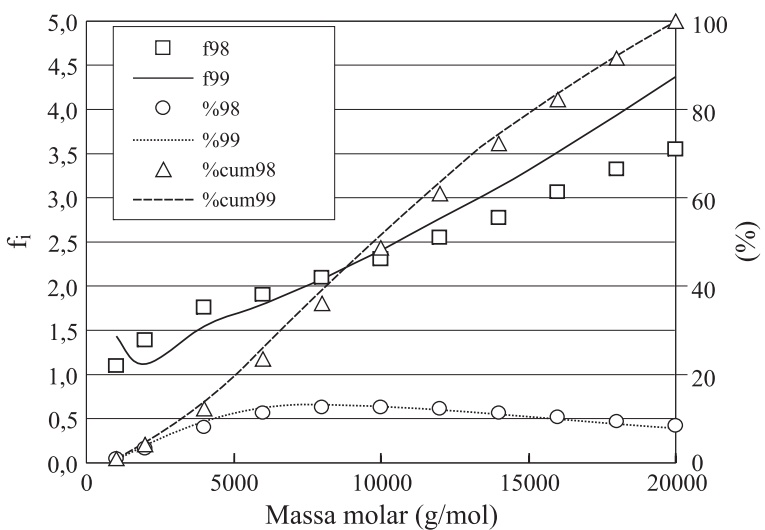

Figura 1. Curvas $f_{i} x M M, \% x M M$ e \%cumxMM (HTPB/ISOFEN 98 e 99)

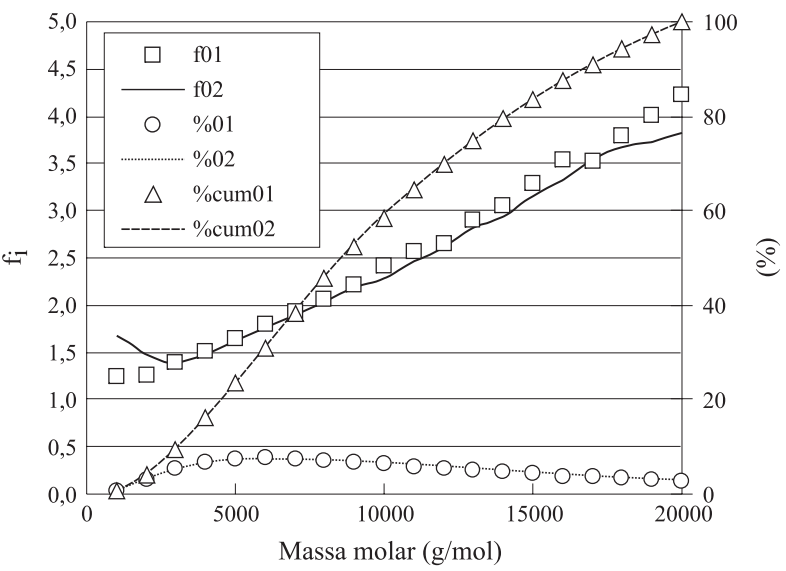

Figura 2. Curvas $f_{i} x M M, \% x M M$ e \%cumxMM (HTPB-ISO/01 e 02-2000) 


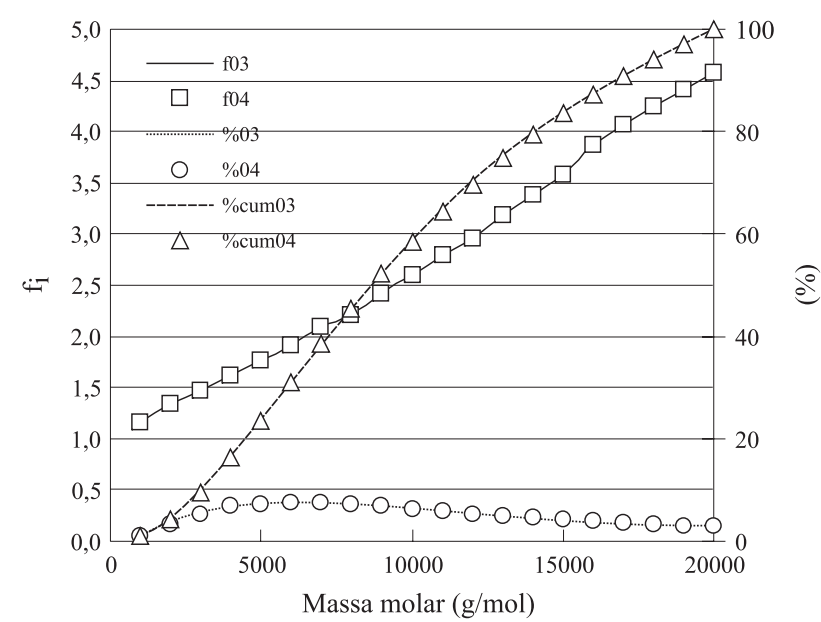

Figura 3. Curvas $\mathrm{f}_{\mathrm{i}} \mathrm{xMM}, \% \mathrm{xMM}$ e \%cumxMM (HTPB-ISO/03 e 04-2000)

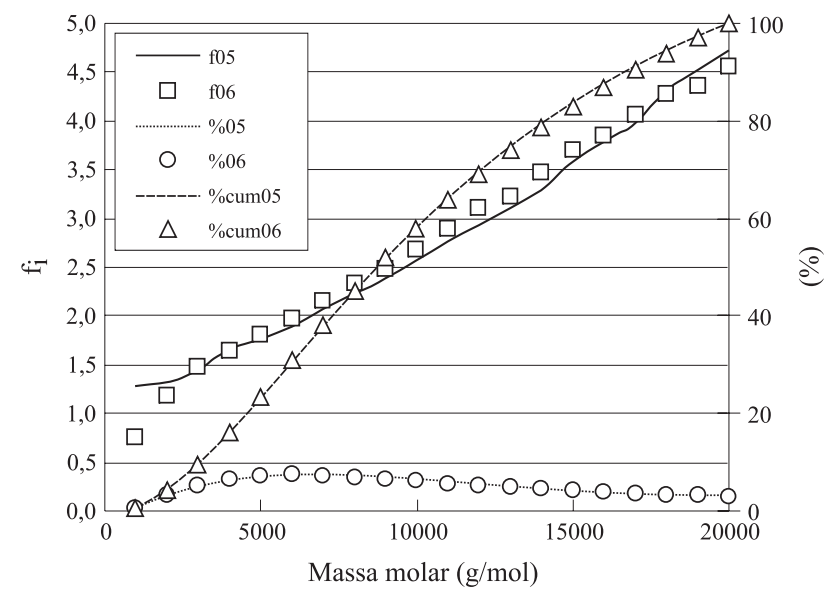

Figura 4. Curvas $\mathrm{f}_{\mathrm{i}} \mathrm{xMM}, \% \mathrm{xMM}$ e \%cumxMM (HTPB-ISO/05 e 06-2000)

(\%cumxMM) em função da distribuição de massa molar e a distribuição da funcionalidade com a massa molar $\left(\mathrm{f}_{\mathrm{i}} \mathrm{xMM}\right)$ obtidas para os vários lotes de HTPB derivatizados.

Estas figuras mostram que os resultados obtidos para as amostras derivadas do mesmo lote de HTPB são bastante repetitivos, indicando que o processo de derivatização está bem controlado.

Em todos os casos a funcionalidade aumentou com o aumento da massa molar, como esperado para HTPB obtido via radical livre ${ }^{[1,2]}$, sendo que os valores de $f_{i}$ estão na mesma faixa dos obtidos por Ninan et al ${ }^{[3]}$.

A Figura 5 compara as curvas de funcionalidade das amostras. Pode ser observado que a funcionalidade é um pouco maior quando a $[\mathrm{OH}]$ é maior (Tabela 1 ), ou seja, o método SEC é sensível para detectar diferenças entre os lotes de HTPB.

$\mathrm{Na}$ Tabela 3 pode ser visto mais claramente o teor de cadeias não participantes da reticulação $\left(\mathrm{f}_{\mathrm{i}} \leq 1\right)$, cadeias extensoras $\left(1<\mathrm{f}_{\mathrm{i}}<3\right)$ e cadeias reticuladoras $\left(\mathrm{f}_{\mathrm{i}} \geq 3\right)$ para cada lote, obtidos a partir das Figuras 1 a 4, os quais são coerentes com o valor de $[\mathrm{OH}]$ (Tabela 1 ), ou seja, quanto menor o valor de $[\mathrm{OH}]$ maior foi o teor de $\left(1<\mathrm{f}_{\mathrm{i}}<3\right)$. fn foi calculada como a média aritmética das funcionalidades individuais.

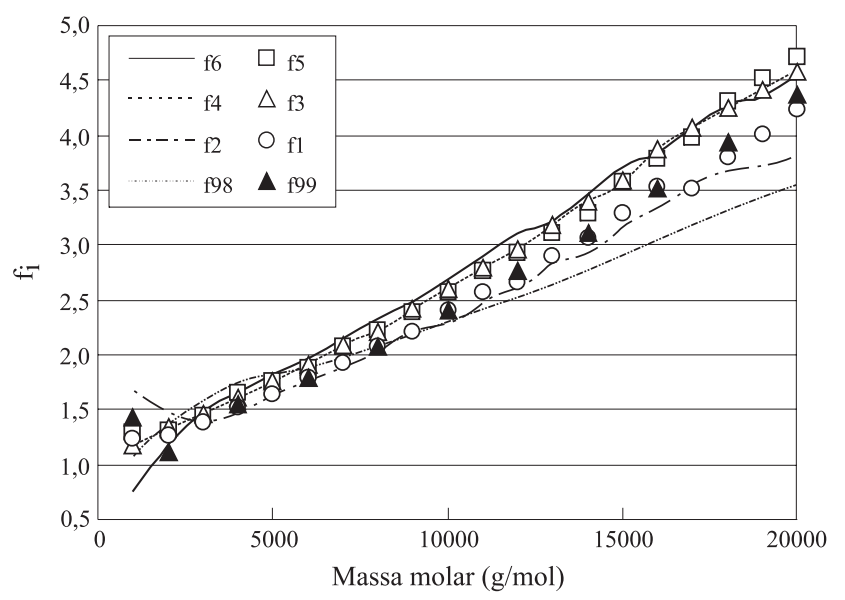

Figura 5. Comparação entre as curvas $f_{i} x M M$ de todos os derivados (Tabela 1)

Tabela 3. Teor de $f_{i} \leq 1,1<f_{i}<3$ e $f_{i} \geq 3(\%)$

\begin{tabular}{ccccc}
\hline Amostra & $\mathbf{f} \leq \mathbf{1}$ & $\mathbf{1}<\mathbf{f}<\mathbf{3}$ & $\mathbf{f} \geq \mathbf{3}$ & $\mathbf{f n}$ \\
\hline HTPB/ISOFEN 98 & 0 & 72 & 28 & 2,35 \\
HTPB/ISOFEN 99 & 0 & 59 & 41 & 2,55 \\
HTPB-ISO/01-2000 & 0 & 75 & 25 & 2,55 \\
HTPB-ISO/02-2000 & 0 & 79 & 21 & 2,49 \\
HTPB-ISO/03-2000 & 0 & 70 & 30 & 2,78 \\
HTPB-ISO/04-2000 & 0 & 70 & 30 & 2,78 \\
HTPB-ISO/05-2000 & 0 & 69 & 31 & 2,78 \\
HTPB-ISO/06-2000 & 0 & 64 & 36 & 2,80 \\
\hline
\end{tabular}

\section{Ensaios de tração}

As condições de cura dos $\mathrm{PU}\left(8\right.$ dias a $50{ }^{\circ} \mathrm{C}$ ) foram estabelecidas com base em experiência anterior com este tipo de material ${ }^{[13]}$. A verificação de que os PU estavam totalmente curados foi feita através de análise FT-IR de filme líquido dos extratos dos PU, não sendo observada a presença de isocianato residual.

A Figura 6 apresenta a comparação entre as curvas de tração versus alongamento das três amostras de PU e a Tabela 4 relaciona os valores de índice de hidroxilas e distribuição de funcionalidade das amostras de HTPB com os dados dos ensaios de tração dos respectivos PU.

Analisando os dados da Tabela 4 observa-se que o aumento da funcionalidade leva a um ligeiro aumento na tensão de ruptura e a uma redução mais acentuada no alongamento de ruptura. As diferenças na tensão de ruptura são pouco significativas, situando-se praticamente dentro do desvio padrão das medidas. Por outro lado, os valores de alongamento refletem melhor as diferenças da distribuição de funcionalidade, mesmo estas sendo pequenas, como é o caso das amostras em questão. Este resultado é coerente com o esperado, visto que uma maior funcionalidade leva a um aumento da reticulação da matriz polimérica, aumentando a 


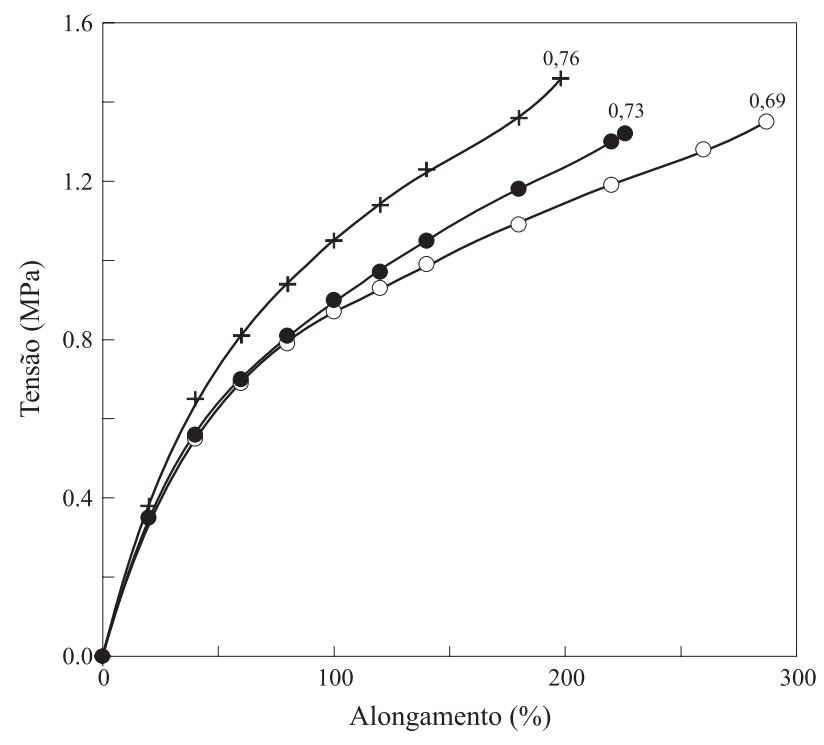

Figura 6. Curvas de tração versus alongamento das três amostras de PU em função do índice de hidroxilas do HTPB.

Tabela 4. Propriedades mecânicas em função da distribuição de funcionalidade

\begin{tabular}{lccc}
\hline \multicolumn{1}{c}{ HTPB } & $\begin{array}{c}\text { Lote 01/98, } \\
\text { Tb 14 }\end{array}$ & $\begin{array}{c}\text { Lote 76/97, } \\
\text { Tb 06 }\end{array}$ & $\begin{array}{c}\text { Lote 73/97, } \\
\text { Tb 10 }\end{array}$ \\
\hline$[\mathrm{OH}](\mathrm{meq} / \mathrm{g})$ & 0,69 & 0,73 & 0,76 \\
$1<\mathrm{F}<3(*)$ & $75 / 79$ & $70 / 70$ & $64 / 69$ \\
$\mathrm{~F} \geq 3(*)$ & $25 / 21$ & $30 / 30$ & $36 / 31$ \\
$\mathrm{~F}_{\mathrm{n}}$ & 2,52 & 2,78 & 2,79 \\
$\sigma \mathrm{r}(\mathrm{MPa})$ & $1,35 \pm 0,04$ & $1,32 \pm 0,06$ & $1,46 \pm 0,09$ \\
$\varepsilon \mathrm{r}(\%)$ & 287 & 226 & 198 \\
\hline
\end{tabular}

${ }^{(*)}$ - valores individuais de 2 determinações

resistência à tração e reduzindo o alongamento do material $^{[2]}$.

Deve ser salientado que é prematuro afirmar se as diferenças observadas com o PU sem carga também seriam observadas caso os ensaios de tração fossem realizados em amostras de PU contendo carga, como é o caso de propelente compósito. Esta questão pode ser esclarecida com futuros trabalhos.

\section{Conclusão}

A utilização do método SEC dupla detecção possibilitou a determinação da distribuição de funcionalidade do Polibutadieno Líquido Hidroxilado Liquiflex P, utilizado em propelentes e proteções térmicas no CTA.

A repetitividade do método foi avaliada pela derivatização de duas alíquotas de cada lote de HTPB, que apresentaram comportamento semelhante.

O método mostrou-se sensível a pequenas diferenças de funcionalidade e sua distribuição podendo ser utilizado para a caracterização mais completa da resina HTPB.
Ensaios de tração realizados em amostras de PU sem carga refletiram as pequenas diferenças de distribuição de funcionalidade existentes entre os lotes de polibutadieno hidroxilado.

\section{Agradecimentos}

Ao CNPq.

\section{Referências Bibliográficas}

1. Panicker, S. S.; Ninan, K. N - Polymer Int., 37(4), p.255-9 (1995).

2. Ramarao, M.; Scariah, K. J.; Ravindran, P. V.; Chandrasekharan, G.; Alwan, S. and Sastri, K. S. - J. Appl. Polym. Sci., 49, p.435-444 (1993).

3. Ninan, K. N.; Balagangadharan, V. P. and Catherine, K. B.Polymer, 32(4), p.628-635 (1991).

4. Law, R. L.- J. Polym. Sci., A1(9), p.589-615 (1971).

5. Muenker, A. H.and Hudson, B. E.- J. Macromol. Sci. Chem.,A3 (7), p.1465-1483 (1969).

6. Chang, M. S.; French, D. M. and Rogers, P. L.- J. Macromol. Sci. Chem., A7 (8), p.1727-1749 (1973)

7. Inagaki, H. and Donkai, N. - J.Appl. Polym. Sci., 29, p.37413752 (1984).

8. Anderson, J. N.; Baczek, S. K.; Adams, H. E. and Viescelius, L. E. - J. Appl. Polym. Sci., 19, p.2255-2267(1975).

9. Baczek, S. K.; Anderson, J. N.; Adams, H. E. - J. Appl. Polym. Sci., 19, p.2269-2277 (1975)

10. Ceccaldi, J. C. and Pohlig, V. -"Distribution des founctions hydroxyles du R45 M par GPC", N.S. No 19/77/CRB/ NP, Societe Nationale des poudres et explosifs (SNPE) - Centre de recherches du Bouchet (1977).

11. Ninan, K. N; Balagangadharan, V. P; Ambikadevi. K. and Catherine, K. B. - "Influence of hydroxyl contents", in: Frontiers of Polymer Research, p.595-599, P.N Prasad \&. P.K. Nigam (ed.), Plenum Press, New York, 1991, New Delhi, India (1991).

12. Panicker, S.S.; Ninan, K.N. - J. Appl. Polym. Sci., 56, p.1797-1804 (1995).

13. Rezende, L. C. -"Envelhecimento de propelente compósito à base de polibutadieno hidroxilado",. Tese de doutorado, Universidade Estadual de Campinas, Brasil (2001).

Enviado: 20/06/05

Reenviado: 25/10/05

Aprovado: 29/11/05 